\title{
La actividad artística como regulador de los procesos de ansiedad y agresividad en niños
}

\begin{abstract}
RESUMEN
Existen investigaciones y publicaciones que indican que la danza es una buena herramienta para ayudar a los niños a regular sus niveles de estrés y a reducir su nivel de ansiedad. El objetivo de esta investigación es el de evaluar comparativamente el efecto de la práctica regular de danza en el control del estrés y la disminución de la ansiedad en niños. Estudio de corte experimental de tipo longitudinal, con muestra no probabilística intencional de 146 niños, 50 niños del grupo experimental que asistieron al programa de danza, 50 niños que practicaban actividad física regular y 46 niños sedentarios. Se aplicaron los cuestionarios CAS, STAIC y el Inventario de Estrés Cotidiano Infantil (IECI). El grupo de danza tuvo el mayor descenso en niveles de estrés y ansiedad. El grupo con ejercicio físico deportivo mostró una tendencia marcada a permanecer constante. En el grupo control de niños sedentarios, sin ningún tratamiento, se halló una notable inclinación hacia el aumento de los niveles de estrés. Los resultados confirman que el estrés puede disminuir con la práctica regular de danza. El ejercicio físico deportivo también reduce el estrés, pero sin la misma estabilidad en los resultados ya que, en algunos casos individuales, aumenta los niveles de ansiedad y estrés.
\end{abstract}

Palabras clave: Educación artística; Danza; Estrés; Ansiedad; Agresividad.

\section{INTRODUCCIÓN}

Existe una amplia bibliografía internacional que hace referencia a los beneficios de la actividad artística. Eisner $(1995,2004)$ habla en sus estudios sobre la importancia del arte y de la educación artística para mejorar la experiencia humana, sus capacidades y el conocimiento del mundo, desarrollando la autonomía, el intelecto y la sensibilidad. En este mismo sentido, García Ríos (2005) indica que la educación artística favorece el desarrollo total del ser humano, de aptitudes y actitudes sensitivas, creativas, cognitivas, expresivas y prácticas.
María Esther Pérez

Peláez ${ }^{i}$

Universidad

Internacional de

Valencia, España

Amador Cernuda Lago ii Universidad Rey Juan Carlos, España 
Teniendo en cuenta la importancia del arte dentro de la educación integral del ser humano, diferentes organismos internacionales se han ocupado del desarrollo de políticas en materia de educación artística. La UNESCO, en su hoja de ruta para la educación artística (2006), señala la importancia de la educación artística para el desarrollo de capacidades individuales, la mejora de la calidad de la educación, el fomento de la expresión y la comunicación de la diversidad cultural.

Como indica Esquivel Cruz (2018):

La Organización de las Naciones Unidas para la Educación, la Ciencia y la Cultura (UNESCO, 1990) ha sido la fuerza motriz del desarrollo de iniciativas políticas en materia de educación y cultura. La presencia del arte en la educación, a través de la educación artística y la educación por el arte, contribuye de manera decisiva al desarrollo integral de los niños y jóvenes, al tiempo que impulsa el diálogo y la cohesión social. (p. 8)

Estudios científicos indican que la danza es una buena herramienta para regular estados y procesos físicos y mentales. San-Juan-Ferrer e Hípola (2020) encuentran en su investigación que la danza es un elemento favorecedor de diferentes aspectos de la inteligencia emocional y es un componente beneficioso para el desarrollo emocional y afectivo del ser humano. Como muestran diferentes investigaciones, la danza puede aportar beneficios emocionales, físicos, sociales, psicológicos y espirituales (Quiroga Murcia et al., 2010); fomenta la creatividad (Mc. Donald, 1991); puede mejorar la actitud hacía los procesos de aprendizaje (Stinson, 1997); mejora el dolor, la depresión, los síntomas cognitivos y los trastornos de sueño en mujeres con fibromialgia (Marín et al., 2019); actúa sobre el deterioro de las facultades mentales y la demencia senil (Verghese et al., 2003); aporta beneficios emocionales a personas con demencia reduciendo la tensión, la depresión y la confusión (Andrés Terán et al., 2019); y puede ser efectiva a la hora de mejorar la salud autoevaluada en adolescentes, mejorando problemas como la ansiedad, la depresión o la fatiga (Duberg et al., 2013).

\section{MARCO TEÓRICO}

El ritmo de vida actual y los constantes cambios a nivel socioeconómico, cultural y tecnológico, unidos a situaciones personales, pueden provocar estrés, ansiedad y conductas de agresividad en sectores de la población vulnerables, en particular entre aquellos grupos más sensibles, los de los niños y adolescentes. Desde el ámbito científico, se intenta dar respuesta a los problemas que afectan a la salud y la calidad de vida de los más jóvenes. La agresividad y violencia en niños se relaciona con conductas disruptivas, que pueden desembocar en problemas como estrés, ansiedad, abandono escolar, depresión y comportamientos de riesgo (Díaz et al., 2013; Gumpel \& Meadan, 2000; Henao Escobar, 2005). Pinheiro (2006), en el informe mundial contra la violencia en niños y niñas de la UNICEF, alerta sobre la gravedad del problema y las 
enormes consecuencias que puede tener en los niños, al tratarse de individuos cuyo sistema nervioso y neurológico se encuentra en pleno desarrollo.

Se ha contrastado que diversos comportamientos asociados a la agresión dependen de factores ambientales y familiares que son principalmente aprendidos en las edades tempranas (Díaz Aguado, 2005; Gallego Henao, 2011; Tremblay et al., 2004). Estos pueden igualmente ser regulados y modificados a través de la educación. Como indica Pinheiro (2006), "la prevención de la violencia contra los niños y niñas requiere cooperación y colaboración entre muchos aliados. En efecto, prevenir y responder a la violencia contra los niños debería ser asunto de todos" (p. 5).

Como afirma Henao Escobar (2005), los problemas de comportamiento agresivo se deben tratar lo antes posible, permitiendo el correcto desarrollo físico, psíquico y socioemocional:

Dado que con frecuencia los problemas de comportamiento agresivo se comienzan a externalizar en los años pre-escolares, y teniendo en cuenta que es en este período en el que se produce la socialización de la agresión, resulta muy conveniente actuar en este ciclo de la vida de tal manera que se frene tempranamente el desarrollo de una trayectoria hacia la violencia. Además, si se tiene presente que durante los años pre-escolares los niños presentan avances notorios en su desarrollo cognitivo, lingüístico y socioemocional, en este período presentan condiciones óptimas para desarrollar competencias sociales, cognitivas y emocionales que sirvan como factores protectores contra el desarrollo o la continuación de patrones de comportamiento agresivo. (p. 171)

Los estudios realizados por Fariñas en Cuba con niños hiperactivos (Cernuda, 2012, 2015; Fariñas, 1999, 2004) y replicados en Suecia (Grönlund et al., 2005) muestran que la danza puede ser una excelente herramienta para mejorar el comportamiento y el rendimiento de los niños en clase, y que no solo actúa sobre los niveles de atención y concentración, sino que también mejora los niveles de agresividad de los niños de entre 7 y 9 años.

Nuestra investigación de corte experimental, realizada en España, se centra en el estudio de los niveles de estrés y ansiedad en niños de 7 a 13 años. La mejora de estos niveles se asocia con una mejor calidad de vida, mejor salud física y psíquica, mejor rendimiento escolar y niveles más bajos de agresividad.

Numerosos estudios en distintas áreas geográficas y culturas muy diferenciadas muestran la relación entre la práctica de la danza y la reducción del estrés y la ansiedad. Grasser et al. (2019) realizaron una intervención con niños sirios refugiados utilizando la danza para trabajar el trauma y el afrontamiento del estrés y la ansiedad, logrando resultados positivos en la reducción de la ansiedad y las formas de enfrentar el trauma involucrado en los procesos de sufrimientos de niños refugiados y observando mejoras en su salud mental. Weitz y Opre (2019) realizaron en Rumania un estudio que investigó el efecto combinado de danza con terapia cognitiva en la eficacia del tratamiento para niños con trastorno de ansiedad (EA), y los resultados 
revelaron una reducción de ansiedad. Vinesett et al. (2017) refieren aspectos muy validos de las danzas africanas en la reducción de la ansiedad y el afrontamiento del estrés. El estudio realizado en Rusia por Izarovskaia et al. (2020) muestra como mediante la práctica de danza niñas de edad entre los 7-8 años de edad muestran un incremento de sus capacidades psicológicas con mayor control de numerosas variables, y entre ellas la ansiedad.

\section{MÉTODO}

Objetivo: Evaluar comparativamente el efecto de la práctica regular de danza en el control del estrés y la disminución de la ansiedad en niños.

Hipótesis: La práctica de la danza mejora los niveles de estrés y ansiedad en los niños.

La danza es una herramienta educativa que colabora significativamente en el control del estrés y la reducción de la ansiedad, frente a la actividad física deportiva y al sedentarismo en niños.

Sujetos: El estudio se realizó con una muestra no probabilística intencional de 146 niños (74 niñas y 72 niños) divididos en tres grupos:

50 niños que practicaban danza (dos sesiones semanales de una hora);

50 niños que practican actividad física deportiva (dos horas semanales de actividad física deportiva);

46 niños sedentarios sin ningún tipo de actividad física ni deportiva, ni artística.

Los niños practicantes de danza realizaron la actividad en el contexto extraescolar: en el colegio, en academias privadas de baile o escuelas municipales. Las técnicas específicas estudiadas principalmente fueron: danza clásica y danza contemporánea, pero la danza en este contexto se entiende desde un punto de vista pragmático, como lenguaje artístico que utiliza el cuerpo y el movimiento como forma de expresión.

Los niños tenían de 7 a 13 años, edad media de 9 años $(\mathrm{Sd}=3,128)$.

Instrumentos: Se utilizaron los cuestionarios CAS, Cuestionario Ansiedad Infantil (Gillis, 2011), STAIC, Cuestionario de Autoevaluación Ansiedad Estado/Rasgo en Niños (Spielberger, 1990) y el Inventario de Estrés Cotidiano Infantil, IECI (Trianes et al., 2011).

Los cuestionarios fueron seleccionados por su fiabilidad test-retest, por su consistencia interna, y por evidencias externas de validez.

Procedimiento: Los tests se pasaron al principio del curso, y se volvieron a pasar al finalizar el curso (de octubre de 2017 a junio de 2018). Una vez recogidos todos los datos se realizaron distintos análisis estadísticos con el programa SPSS versión 19.0 para Windows. Se realizaron los siguientes contrastes.

- Análisis de frecuencias de la muestra, que nos indica el porcentaje de sujetos que puntúan en los distintos valores de cada variable.

- Análisis descriptivo, que nos da los valores máximo y mínimo de cada variable, su media y su desviación típica. 
Estos dos análisis permiten describir la muestra con la que estamos trabajando.

Diferencia de medias, que nos permitirá observar si existen diferencias significativas en las medidas de las variables.

\section{RESULTADOS}

Una vez analizados los controles de los resultados, indican que existen diferencias significativas entre las puntuaciones obtenidas entre los estudiantes que practicaban danza, ejercicio deportivo y los que no realizaban ninguna actividad física, deportiva o artística (ver Tabla 1 y Figura 1). En la variable Ansiedad Estado ( $\mathrm{p}<.01)$, presentan mayores puntuaciones los niños que no tenían ningún tipo de actividad física, deportiva, o artística. En los niños del grupo sedentario sin ningún tratamiento de actividad ni deportiva ni artística, se ha observado una tendencia al aumento de los niveles de estrés y ansiedad. El grupo de niños con ejercicio físico deportivo mostró una tendencia a permanecer constante con leves incrementos y algunas reducciones de los niveles. El grupo de los niños con actividad de danza tuvo las mayores reducciones de ansiedad, mostrando ausencia de problemas de estrés y ansiedad.

Tabla 1

Resultados Estadísticos de los Grupos de Investigación

\begin{tabular}{l|l|l|l|l|l}
\hline Grupo & Controles & $\mathrm{N}$ & Media & Desviación típica & $\begin{array}{l}\text { Error típico } \\
\text { de la media }\end{array}$ \\
\hline \multirow{2}{*}{ Sedentarios } & Control 1 $^{\circ}$ & 46 & 63,20 & 28,617 & 3,160 \\
\cline { 2 - 6 } & Control 2 $^{\circ}$ & 46 & 65,75 & 27,068 & 3,283 \\
\hline \multirow{2}{*}{$\begin{array}{l}\text { Actividad física } \\
\text { deportiva }\end{array}$} & Control 1 $^{\circ}$ & 50 & 63,13 & 23,935 & 2,903 \\
\cline { 2 - 6 } & Control 2 $^{\circ}$ & 50 & 51,49 & 27,193 & 3,003 \\
\hline \multirow{2}{*}{ Danza } & Control 1 & 50 & 62,90 & 23,703 & 2,874 \\
\cline { 2 - 6 } & Control 2 & 50 & 50,16 & 27,091 & 2,992 \\
\hline
\end{tabular}

Fuente: Elaboración propia a través de los datos obtenidos en el estudio. 
Figura 1

Niveles de Estrés y Ansiedad en los Tres Grupos de Estudio: Sedentarios,

Actividad Física Deportiva y Danza

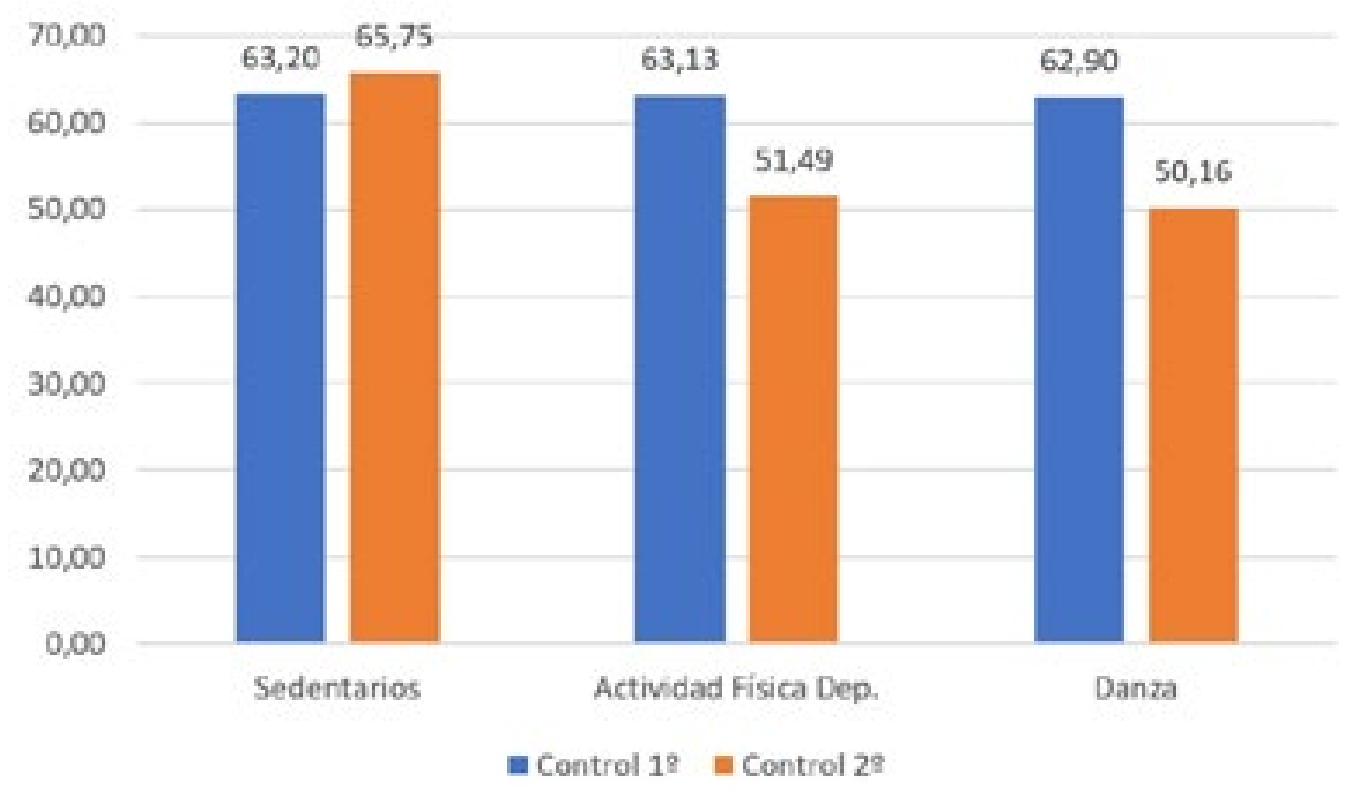

Fuente: Elaboración propia a través de los datos obtenidos en el estudio.

\section{DISCUSIÓN Y CONCLUSIONES}

En base a los resultados de esta investigación experimental, podemos afirmar que la danza es una buena herramienta para mejorar estados de estrés y ansiedad en niños. Se contrastan positivamente nuestras hipótesis principales: H1: La práctica de la danza mejora los niveles de estrés y ansiedad en los niños, y H2: La danza es una herramienta educativa que colabora significativamente en el control del estrés y la reducción de la ansiedad, frente a la actividad física deportiva y al sedentarismo en niños. Hemos contrastado que la actividad física deportiva también colabora en el proceso de reducir los niveles de estrés y ansiedad en niños, en concordancia con otros estudios como el de Remor y Pérez Llantada Rueda (2007), sin embargo, los resultados no presentan la misma estabilidad ni robustez, como se indica en Tabla 1, ya que, en algunos casos particulares, se ha detectado en nuestro estudio un incremento de los niveles de ansiedad y estrés en algunos niños. Estos resultados nos hacen cuestionar si el aumento de estos parámetros en algunos niños que practican actividad física deportiva se debe a los niveles de competitividad y agresividad que se trasmiten en la enseñanza y práctica de algunos deportes, incluso en estas edades tempranas, como factor desencadenante de esta variabilidad. En este sentido, algunos estudios muestran cómo la competencia deportiva puede aumentar los niveles de ansiedad en deportistas (Ries et al., 2012) y causar niveles moderados de estrés en niños (Caputo et al., 2017; Rose Junior, 1998). 
Con respecto a los niños sedentarios, son los que muestran el mayor incremento en los niveles de estrés y ansiedad, con un aumento de 2,55 puntos en la media del segundo test (ver Tabla 1). Este estudio nos facilita como investigadores de las artes y el deporte, en particular de la danza y la actividad física, en la identificación de las ventajas comparativas que esta tiene en la reducción de los niveles de ansiedad y estrés, y consecuentemente en la mejora de la calidad de vida y la salud, de los niños que practican estas disciplinas frente a aquellos sin actividades similares.

Es posible que la ventaja psicopedagógica de la danza en la mitigación y corrección de los problemas relacionados con el estrés, la ansiedad y la agresividad es que tiene primero un componente de actividad física, no competitivo a esta edad temprana, pero también un componente fundamentalmente de actividad artística. En este sentido, enseña a los niños que la practican un lenguaje que les permite expresarse y comunicarse de forma diferente, pero prácticamente innata en cada uno de ellos, y les ayuda a comprender mejor sus sentimientos, descubrir y conocer su propio cuerpo y las posibilidades de movimiento que ofrece y desarrollar la empatía. Algunos de los sujetos de nuestro estudio han comunicado en sus clases, que la danza "les hacía sentir libres", que "al bailar se olvidaban de sus problemas y las cosas malas", que gracias a la danza "pueden jugar con niños que antes no conocían", entre otras afirmaciones espontáneas a la pregunta de que sienten al hacer danza. Martin et al. (2018) realizaron una revisión en la que incluyeron 37 estudios científicos de los que el 81,1\% informaron de una reducción significativa de los niveles de estrés de los participantes debido a la intervención con modalidades artísticas, destacando el papel danza.

Si a todos los beneficios de la actividad artística en general y de la danza en particular le añadimos el descenso de los niveles de estrés y ansiedad, junto con una mejor regulación en los niveles de agresividad, podemos hablar de una actividad de gran valor para la salud física, psíquica y emocional, que debe seguir siendo promovida por organismos internacionales como la UNESCO, y por diferentes sectores públicos y privados de la sociedad.

Es relevante señalar, igualmente, que seguimos detectando una problemática de género en el acceso efectivo a la educación en danza para una parte de la población a la que se le viene históricamente marginando de esta actividad educativa, los niños varones. Ha sido complicado conseguir una distribución proporcional en cuanto a géneros (varones/mujeres) en el grupo que practicaba danza, frente al grupo sedentario y al grupo que practicaba deporte. Esta desigualdad en la distribución de género en una actividad tan beneficiosa como la danza discrimina a una parte importante de la población infanto-juvenil, por motivos sociales y culturales. Los beneficios físicos, sociales y psicológicos deben estar al alcance de todos. En Grecia, Filippou et al. (2018) realizaron un estudio en el que comprobaron que la enseñanza de danzas griegas tradicionales a 131 participantes de ambos sexos incrementaba los niveles de autoconfianza más en los hombres qué en las mujeres, y en ambos sexos se reducían los niveles de ansiedad. Entendemos que es muy importante que niños y niñas tengan los mismos derechos de acceso a cualquier tipo de actividad artística, estableciendo programas de información a pedagogos, padres y niños sobre los beneficios contrastados que aporta la educación 
artística, y en particular la danza. Como profesores e investigadores, como científicos de la pedagogía y de las artes, debemos ayudar en la corrección de esta desigualdad de género existente en la práctica de la danza en algunos países de nuestro entorno cultural. La sociedad del siglo XXI debe estar informada y ser consciente que la discriminación entre actividades por razones tal vez "culturales decimonónicas" basadas en estereotipos de género que establecen unos patrones hacen que la danza y sus virtudes aquí detectadas no permeen entre los varones de nuestra sociedad con la misma profusión que entre las mujeres.

A la luz de los datos reflejados en nuestra investigación, recomendamos la práctica de la danza a niños y niñas por igual, por sus cualidades, y por ser una magnífica herramienta para disminuir los estados de estrés, ansiedad y agresividad, y por lo tanto mejorar la salud y la calidad de vida de los más pequeños. La danza es a la luz de la evidencia científica ese magnífico "superalimento", mejor dicho "super instrumento artístico cultural" que permite ayudar a desarrollar a nuestros niños la mejor versión de sí mismos, regulando los niveles de estrés, ansiedad y agresividad que pudieran tener.

Esta investigación es consecuente con la línea de trabajo donde proponemos el abordaje de nuevas investigaciones relacionadas con la educación artística, la danza y la regulación de procesos y estados mentales en niños y jóvenes a través del arte. Nuestra investigación presenta limitaciones relativas a la muestra de alumnos y el periodo de investigación limitado a un año. Entendemos que este estudio y similares deben ser replicados con el objeto de detectar los efectos aquí indicados, no solamente en otros contextos culturales y sociales, sino en otros grupos de edad y países en próximos años. De esta manera se podrá reforzar la evidencia científica y seguir trabajando en la detección de relaciones causa-efecto sobre el aprendizaje de la danza y las disciplinas artísticas.

En estos contextos de investigación, docencia en la Universidad, enseñando y aprendiendo a enseñar con nuestros alumnos, en la práctica de las disciplinas artísticas y su investigación científica, nos encontraremos en los próximos años.

\section{REFERENCIAS}

Andrés Terán, A. L., Pérez Sáez, E., Cernuda Lago, A., \& Sánchez Vázquez, R. (2019). Propiedades psicométricas del Profile of Mood States (POMS) en personas con demencia y su aplicación en la evaluación de los efectos de la danza creativa terapéutica. Revista de Neurología, 68(5), 190-198. https://doi.org/10.33588/ rn.6805.2018266

Caputo, E. L., Rombaldi, A. J., \& Silva, M. C. (2017). Sintomas de estresse pré-competitivo em atletas adolescentes de handebol. Revista Brasileira de Ciências do Esporte, 39(1), 68-72. https://doi.org/10.1016/j.rbce.2016.01.006

Cernuda, A. (2012). Arte social comunitario y aplicaciones clínicas del psicoballet. 40 años de experiencia cubana. Actas del Congreso Internacional de Intervención Social, Arte Social y Arteterapia (pp. 1-11). Universidad de Murcia. 
Cernuda, A. (2015). Efectos de un programa de psicoballet en la mejora de la atención en niños hiperactivos. Proceedings of 3rd International Congress of Educational Sciences and Development (pp. 20-24). Asociación Española de Psicología Conductual (AEPC).

Díaz Aguado, M. J. (2005). Por qué se produce la violencia escolar y como prevenirla. Revista Iberoamericana de Educación, 37, 17-47.

Díaz Aguado, M. J., Martínez Arias, R., \& Ordóñez, A. (2013). Prevenir la drogodependencia en adolescentes y mejorar la convivencia desde una perspectiva escolar ecológica. Revista de Educación, Extr. 1, 338-362. https://doi. org/10.4438/1988-592X-RE-2013-EXT-251

Duberg, A., Hagberg, L., Sunvisson, H., \& Möller, M. (2013). Influencing self-rated health among adolescent girls with dance intervention: A randomized controlled trial. JAMA Pediatrics, 167(1), 27-31. https://doi.org/10.1001/ jamapediatrics.2013.421

Eisner, E. W. (1995). Educar la visión artística. Paidós.

Eisner, E. W. (2004). El arte y la creación de la mente: El papel de las artes visuales en la transformación de la conciencia. Paidós.

Esquivel Cruz, M. C. (2018). El proceso de integración de los niños a los talleres de creación artística de teatro y danza en la Casa de Cultura Pedro Junco, de Pinar del Río, Cuba. IE Revista de Investigación Educativa de la REDIECH, 9(16), 7-27.

Fariñas, G. (1999). Temas de psicoballet. Hospital Psiquiátrico de La Habana.

Fariñas, G. (2004). Psicoballet, teoría y práctica en Cuba y Puerto Rico. Universidad de Puerto Rico.

Filippou, F., Rokka, S., Pitsi, A., Gargalianos, D., Bebetsos, E., \& Filippou, D. (2018). Interdisciplinary Greek traditional dance course: Impact on student satisfaction and anxiety. International Journal of Instruction, 11(3), 363-374. https://doi.org/10.12973/iji.2018.11325a

Gallego Henao, A. M. (2011). La agresividad infantil: Una propuesta de intervención y prevención pedagógica desde la escuela. Revista Virtual Universidad Católica del Norte, 33, 295-314.

García Ríos, A. S. (2005). Enseñanza y aprendizaje en la educación artística. El Artista, 2, 80-97.

Gillis, J. S. (2011). Cuestionario ansiedad infantil. TEA Ediciones.

Grasser, L. R., Al-Saghir, H., Waanna, C., Spinei, J., \& Javanbakht, A. (2019). Moving through the trauma: Dance/movement therapy as a somatic-based intervention for addressing trauma and stress among Syrian refugee children. Journal of The American Academy of Child \& Adolescent Psychiatry, 58(11), 1124-1126. https://doi.org/10.1016/j.jaac.2019.07.007

Grönlund, E., Barbro, R., \& Weibull,J. (2005). Dance/movement therapy as an alternative treatment for young boys diagnosed as ADHD: A pilot study. American Journal of Dance Therapy, 27(2), 37-85.

Gumpel, T. P., \& Meadan, H. (2000). Children's perceptions of schoolbased violence. British Journal of Educational Psychology, 70(3), 391-404. https://doi. org/10.1348/000709900158191

Henao Escobar, J. (2005). La prevención temprana de la violencia: Una revisión de programas y modalidades de intervención. Universitas Psychologica, 4(2), 161-177. 
Izarovskaia I. V., Smirnova L. V., Sumak, E. N., \& Izarovskaia, O. B. (2020). Fitness dance for developing basic movement qualities in preschool children and improving their psychic status. Human. Sport. Medicine, 19(1), 86-92. https://doi. org/10.14529/hsm190112

Mc. Donald, C. J. (1991). Creative Dance in Elementary Schools: A Theoretical and Practical Justification. Canadian Journal of Education, 16(4), 434-441.

Marín, F., Colina, E., \& Duque, I. L. (2019). Danza terapéutica y ejercicio físico. Efecto sobre la fibromialgia. Hacia la Promoción de la Salud, 24(1), 17-27. https://doi.org/10.17151/hpsal.2019.24.1.3

Martin, L., Oepen, R., Bauer, K., Nottensteiner, A., Merghein, K., Gruber, H., $\&$ Koch, S. (2018). Creative arts interventions for stress management and prevention - A systematic review. Behavioral Sciences, 8(2), 28-39. https://doi.org/10.3390/ bs 8020028

Pinheiro, P. S. (2006). Informe sobre la violencia contra niños y niñas. UNICEF. https://violenceagainstchildren.un.org/sites/violenceagainstchildren. un.org/files/document_files/world_report_on_violence_against_children_sp.pdf

Quiroga Murcia, C., Kreutz, G., Clift, S., \& Bongard, S. (2010). Shall we dance? An exploration of the perceived benefits of dancing on well-being. Arts \& Health, 2(2), 149-163. https://doi.org/10.1080/17533010903488582

Remor, E., \& Pérez Llantada Rueda, M. C. (2007). La relación entre niveles de la actividad física y la experiencia de estrés y de síntomas de malestar físico. Interamerican Journal of Psychology, 4(3), 313-322.

Ries, F., Castañeda Vázquez, C., Campos Mesa, M. C., \& Castillo Andrés, O. (2012). Relaciones entre ansiedad-rasgo y ansiedad-estado en competiciones deportivas. Cuadernos de Psicología del Deporte, 12(2), 9-16. https://doi.org/10.4321/ s1578-84232012000200002

Rose Junior, D. de (1998). Lista de sintomas de "stress" précompetitivoinfanto-juvenil: Elaboração e validação de um instrumento. Revista Paulista de Educação Física, 12(2), 126-133. https://doi.org/10.11606/issn.25945904.rpef.1998.139540

San-Juan-Ferrer, B., \& Hípola, P. (2020). Emotional intelligence and dance: A systematic review. Research in Dance Education, 21(1), 57-81. https://doi.org/10.108 0/14647893.2019.1708890

Spielberger, C. D. (1990). STAIC Cuestionario de autoevaluación ansiedad estado/rasgo en niños. TEA Ediciones.

Stinson, S. W. (1997). A question of fun: Adolescent engagement in dance education. Dance Research Journal, 29(2), 49-69. https://doi.org/10.2307/1478734

Tremblay, R. E., Nagin, D. S., Séguin, J. R., Zoccolillo, M., Zelazo, P. D., Boivin, M., Pérusse, D., \& Japel, C. (2004). Physical aggression during early childhood: Trajectories and predictors. Pediatrics, 114(1), 43-50. https://doi.org/10.1542/ peds.114.1.e43

Trianes, M. V., Blanca, F. J., Fernández, F. J., Escolar, M., \& Maldonado, E. F. (2011). Manual de inventario de estrés cotidiano infantil. TEA Ediciones.

Verghese, J., Lipton, R. B., Katz, M. J., Hall, C. B., Derby, C. A., Kuslansky, G., Ambrose, A. F., Sliwinski, M., \& Buschke, H. ((2003). Leisure activities and the risk of dementia in the elderly. The New England Journal of Medicine, 348, 2508-2516. https://doi.org/10.1056/NEJMoa022252 
Vinesett, A. L., Whaley, R. R., Woods-Giscombe, C., Dennis, P., Johnson, M., Li, Y., Mounzeo, P., Baegne, M., \& Wilson, K. H. (2017). Modified African ngoma healing ceremony for stress reduction: A pilot study. The Journal of Alternative and Complementary Medicine, 23(10), 800-804. https://doi.org/10.1089/acm.2016.0410

Weitz, N., \& Opre, A (2019). The effectiveness of DMT-CBT combination in anxiety disorders treatment: Therapists perceptions. "Education, Reflection, Development", 6th Edition. The European Proceedings of Social and Behavioral Sciences (v. 63, pp. 600-606). Future Academy. https://doi.org/10.15405/epsbs.2019.06.72 
i Universidad Internacional de Valencia, España.

https://orcid.org/0000-0001-5243-5733

ii Universidad Rey Juan Carlos, España.

https: //orcid.org/0000-0002-5993-4647

Toda a correspondência relativa a este artigo

deve ser enviada para:

María Esther Pérez Peláez

Universidad Internacional de Valencia. Facultad de Educación.

Calle d Pintor Sorolla 21

46002 Valencia. España

mariaesther.perezecampusviu.es

Recebido em 29 de abril de 2019

Aceite para publicação em 11 de setembro de 2020 
The artistic activity as a regulator of anxiety and aggressiveness processes in children

\begin{abstract}
There are research and numerous publications that indicates that dance is a good tool in helping children to regulate their stress levels and to reduce their level of anxiety. To evaluate comparatively the effect of regular dance practice in the control of stress and the reduction of anxiety in children. Longitudinal experimental study with intentional non-probabilistic sample of 146 children, 50 children from the experimental group who attended the dance program, 50 children who practiced regular physical activity and 46 sedentary children. The questionnaires CAS, STAIC and the Daily Stress Inventory for Children (IECI) were applied. The dance group had the greatest decrease in levels of stress and anxiety. The group with sports physical exercise showed a marked tendency to remain constant. In the control group of sedentary children, without any treatment, a remarkable inclination towards increasing stress levels was found. The results confirm that stress can decrease with regular dance practice. Sports physical exercise also reduces stress, but without the same stability in the results since, in some individual cases, it increases the levels of anxiety and stress.
\end{abstract}

Keywords: Artistic education; Dance; Stress; Anxiety; Aggressiveness 


\section{A atividade artística como reguladora dos processos de ansiedade e agressividade em crianças}

\section{RESUMO}

Existem pesquisas e publicações que indicam que a dança é uma boa ferramenta para ajudar as crianças a regular os seus níveis de estresse e reduzir o seu nível de ansiedade. 0 objetivo desta pesquisa é avaliar comparativamente o efeito da prática regular de dança no controle do estresse e redução da ansiedade em crianças. Estudo experimental do tipo longitudinal, com amostra intencional não probabilística de 146 crianças, 50 crianças do grupo experimental que frequentavam o programa de dança, 50 crianças que praticavam atividade física regular e 46 crianças sedentárias. Foram aplicados os questionários CAS, STAIC e o Childhood Stress Inventory (IECI). $\mathrm{O}$ grupo de dança apresentou a maior diminuição nos níveis de estresse e ansiedade. 0 grupo com exercício físico desportivo apresentou tendência acentuada de se manter constante. No grupo controle de crianças sedentárias, sem nenhum tratamento, foi observada uma tendência notável de aumento dos níveis de estresse. Os resultados confirmam que o estresse pode diminuir com a prática regular de dança. 0 exercício físico desportivo também reduz o estresse, mas sem a mesma estabilidade nos resultados, pois, em alguns casos individuais, aumenta os níveis de ansiedade e estresse.

Palavras-chave: Educação artística; Dança; Estresse; Ansiedade; Agressividade. 